\title{
Monitoramento das Práticas Integrativas e Complementares em Minas Gerais: a utilização de um instrumento de apoio aos sistemas de informação
}

Monitoring of Integrative and Complementary Practices in Minas Gerais: the use of an instrument to support information systems

El seguimiento de las Prácticas Complemnatrias en Minas Gerais: el uso de una herramienta de apoyo a los sistemas de informaciónn

Gisele Cazarin ${ }^{1}$; Gelza Matos Nunes²; José Geraldo Martins ${ }^{3}$; Mayla Magalhães de Sousa ${ }^{4}$; Ana Paula Medrado de Barcellos ${ }^{5}$

\section{Resumo}

Objetiva-se, com o presente artigo, relatar o desenvolvimento de estratégias de monitoramento da Política Estadual de Práticas Integrativas e Complementares em Minas Gerais, a PEPIC-MG. A inserção de modalidades das Práticas Integrativas e Complementares (PICS) no SUS nos municípios mineiros remonta aos anos 1990. Embora seu incremento tenha ocorrido após 2004, com a mobilização dos entes federativos nos debates para a construção da Política Nacional de Práticas Integrativas e Complementares (PNPIC). A publicação da Política Estadual de PICS de Minas Gerais foi realizada em 2009, quando já havia sido identificado um contingente expressivo de experiências municipais ofertando PICS, por meio de um diagnóstico situacional. O monitoramento dos dados de oferta do Serviço de PICS é realizado, desde 2014, por meio de um conjunto de informações coletadas em Sistemas oficiais de informação, como também por um instrumento específico, desenvolvido pela Coordenação do programa, o Práticas in Loco (PIL). Esse instrumento tem se mostrado muito útil na medida em que possibilita mapear a diversidade das PICS relatadas e sua variação temporal, além de se constituir num importante instrumento de planejamento e gestão do programa.

Descritores: Terapias Complementares; Avaliação em Saúde; Políticas, Planejamento e Administração em Saúde.

\section{Abstract}

The purpose of the present paper is to present strategies aiming monitoring the State Policy of Complementary and Integrative Practives of Minas Gerais State, the PEPIC-MG. The insertion Integrative and Complementary Practices (PICS) in the SUS in Minas Gerais municipalities dates back to the 1990s, although their increase occurred after 2004 with the mobilization of federal entities in the debates for the construction of the National Policy on Integrative and Complementary Practices (PNPIC). The publication of PEPIC-MG occurred in 2009, when a significant contingent of municipal experiences had already been identified through a situational diagnosis as delivering PICS. The monitoring of the offer data of the PICS Service has been carried out since 2014 through

\footnotetext{
${ }^{1}$ Mestre em Saúde Pública pela Fundação Oswaldo Cruz/FIOCRUZ-PE. Docente e pesquisadora do Instituto de Medicina Integral Prof. Fernando Figueira (IMIP). Rua dos Coelhos, 300, Boa Vista, Recife, PE, Brasil. CEP: 50070-550. E-mail: gcazarin@gmail.com 2 Coordenadora das Práticas Integrativas e Complementares da Secretaria Estadual de Saúde de Minas Gerais - SES-MG. Doutoranda da Faculdade de Medicina da Universidade Federal de Minas Gerais - UFMG

3 Coordenador das Práticas Integrativas e Complementares da Secretaria Estadual de Saúde de Minas Gerais - SES-MG.

4 Diretora de Políticas de Atenção Primária da Secretaria Estadual de Saúde de Minas Gerais - SES-MG.

5 Superintendente de Atenção Primária em Saúde da Secretaria Estadual de Saúde de Minas Gerais - SES-MG.
} 
a set of information collected in information systems and also through a specific instrument developed by the State Coordination of PICS, Práticas in Loco (PIL). This instrument has been shown to be useful due to its simplicity to capture the diversity of PICS reported by municipalities and also their temporal fluctuation.

Key-words: Complementary Therapies; Health Evaluation; Health Policy, Planning and Management.

\section{Resumen}

Se propone, con el presente artículo, relatar el desarrollo de estrategias de monitoración de la Política Estatal de Prácticas Integrativas y Complementarias em el estado de Minas Gerais, la PEPIC-MG. La inserción de las modalidades de las Prácticas Integrativas y Complementarias (PICS) en el Sistema Único de Salud de Brasil (SUS) en los municipios de Minas Gerais se remonta a los años de 1990. Aunque su incremento se produjo después del 2004, com la mobilización de los entes federativos em los debates para la construcción de la Política Nacional de Prácticas Integrativas y Complementarias (PNPIC). La publicación de la Política Estatal de PICS de Minas Gerais se realizó en el 2009, cuando ya se habia identificado un contingente expresivo de experiencias municipales ofreciendo PICS por medio de un diagnóstico situacional. La monitorización de los datos de la oferta del servicio de PICS se realiza, desde el 2014, por medio de un conjunto de informaciones recolectadas en sistemas de información, y también en un instrumento específico desarrollado por la Coordinación del programa, el Prácticas in Loco (PIL). Este instrumento se ha mostrado muy útil em la medida que se posibilita el mapeo de la diversidade de las PICS realatadas y su variación temporal además de constituirse importante instrumento de planificación y gestión del programa.

Palabras-claves: Terapias Complementarias; Evaluación en Salud; Políticas, Planificación y Administración en Salud.

\section{Introdução}

A inserção de práticas de saúde tidas como não convencionais ou 'não hegemônicas', nos sistemas públicos de saúde tem seu início no final dos anos 1970, a partir da Primeira Conferência Internacional de Assistência Primária em Saúde, realizada em Alma Ata, Rússia (1978). Posteriormente, a Organização Mundial de Saúde (OMS) define um lócus institucional e passa a fomentar que estados membros integrem as Medicinas Tradicionais e Medicinais Alternativas e Complementares (TM/CAM) aos sistemas de saúde formais. No Brasil, o tema passa a ter visibilidade e relevância após a Oitava Conferência Nacional de Saúde, realizada em $1986^{1-5}$.

As justificativas para essa inclusão consideram uma série de aspectos, como: a ampliação do acesso, a humanização dos serviços, a desmedicalização dos sintomas, a promoção do cuidado e auto-cuidado, o protagonismo dos usuários e o apoio na prevenção e redução da morbimortalidade por doenças crônicas não transmissíveis, entre outras. Destaca-se o fato de que se trata de recursos terapêuticos que buscam estimular os mecanismos naturais, eficazes e seguros, que auxiliam na preservação e recuperação da saúde, com foco na escuta, no vínculo terapêutico e na integração do 
ISSN 2179-6750

ser humano com o meio ambiente e a sociedade ${ }^{4-8}$.

A maior parte dessas práticas baseia-se numa concepção 'vitalista', entendendo a doença como desequilíbrio da energia vital, com ênfase no estado geral do doente e não na doença, numa perspectiva integradora e não organicista. Assim, as propostas de saúde influenciadas por essa doutrina se caracterizam por serem não intervencionistas, mas focadas no indivíduo, mostrando eficazes na superação do modelo biomédico, excessivamente centrado na doença, na assistência curativa e intervenção medicamentosa ${ }^{9}$.

Para $\mathrm{Luz}^{9}$, as PICS enquadram-se numa categoria, derivada de Weber, de formas de cuidado denominadas de 'racionalidades médicas', que são sistemas médicos complexos os quais incluem cinco dimensões básicas, estruturadas em termos teóricos, práticos e simbólicos e que não chegam a se realizar de forma acabada, por terem a capacidade de serem modificadas historicamente pela ação dos agentes sociais, embasadas por uma cosmologia que expressa a cultura onde se originam. Sendo elas: (1) morfologia humana (anatomia), (2) dinâmica vital humana (fisiologia), (3) doutrina médica: define, em cada sistema, o que é o processo saúde-doença, (4) sistema de diagnose, e (5) sistema terapêutico. O estudo dos meios de cuidado, a partir dessas dimensões, permitiu distinguir entre sistemas médicos complexos, como a biomedicina, a medicina tradicional chinesa (MTC), a medicina ayurveda e a homeopatia, de terapias ou métodos de diagnóstico, como: florais de Bach, reiki, iridologia, entre outros ${ }^{8}$.

A inserção de Práticas Integrativas e Complementares (PICS) no Sistema Único de Saúde (SUS) dos municípios mineiros remonta aos anos 1990. Embora seu incremento tenha ocorrido após 2004, com a mobilização dos entes federativos nos debates para a construção da Política Nacional de Práticas Integrativas e Complementares (PNPIC). No diagnóstico situacional realizado, em 2008, durante a fase de proposição de uma Política Estadual de PICS para o estado de Minas Gerais (PEPIC-MG), de 389 municípios, correspondente a cerca da metade (45,6\%) dos municípios do Estado (853), que responderam ao questionário enviado, 76 apontaram a existência de alguma prática, revelando um contingente significativo como ponto de partida. A publicação da PEPIC-MG ${ }^{10}$ ocorreu em 2009, contemplando as abordagens presentes na PNPIC: plantas medicinais/fitoterapia, homeopatia, MTC/acupuntura, medicina antroposófica e termalismo/crenoterapia. Cumpre-nos destacar que tais práticas, de acordo com a mencionada política, devem ser prioritariamente, ofertadas a partir de serviços de atenção primária a saúde (APS), sua regulamentação prevê incentivo financeiro estadual voltado à sua implantação nos municípios mineiros ${ }^{10}$.

O mencionado diagnóstico ${ }^{10}$ também revelou que o estado apresentava cenário marcado pela presença de grande diversidade de PICS. Seguindo a tendência observada nacionalmente, as 
ISSN 2179-6750

principais abordagens presentes nos municípios mineiros, ordenadas por magnitude, eram: a fitoterapia, a homeopatia e a acupuntura, correspondendo juntas por mais da metade da frequência. Além dessas, foram relatadas experiências relativas às seguintes práticas corporais: Tuiná, Shiatsu, Thai Chi Chuan, Lian Gong, Qi Kung, automassagem, meditação, yoga, e ainda um grupo denominado de 'outras', composto por atividades classificadas como integrativas/práticas corporais, a exemplo da: caminhada, reflexologia podal, ginástica laboral, geoterapia, florais, auriculoacupuntura, quiropraxia, reeducação postural global (RPG) e atividade física direcionada à população idosa. No intuito de implantar, ampliar e fortalecer a oferta dos serviços de PICS junto aos municípios, a Coordenação de Práticas Integrativas e Complementares Coordenação Estadual de PICS (CPIC-MG) promoveu cursos de formação, assim como distribuiu insumos voltados à acupuntura e auriculoterapia ${ }^{10}$.

Em 2014, cinco anos após a publicação da PEPIC-MG, 146 municípios mineiros relataram a presença de PICS ${ }^{11}$ (Figura 1), dos quais três haviam avançado na elaboração e publicação de Política Municipal de PICS, a saber: Nova Lima, Santa Rita de Jacutinga e São João Del Rei. A capital do Estado, Belo Horizonte, participou do cenário de institucionalização com a implantação, em 1994, de relevante experiência, denominada de Programa de Homeopatia, Acupuntura e Medicina Antroposófica (PROHAMA) ${ }^{11-13}$.

Os 853 municípios do Estado são distribuídos, do ponto de vista sanitário e administrativo, em 28 Unidades Regionais de Saúde (URS), encarregadas de promover a descentralização/desconcentração das ações. O fluxo de comunicação entre os municípios e o nível central passa preferencialmente pela URS. Em cada uma das URS há um Núcleo de APS, que inclui uma referência técnica em PICS (Figura 1). A CPIC-MG foi criada em 2007, e atualmente está incluída na Superintendência de Ações Primárias de Saúde, oferecendo orientação técnica e suporte às URS no campo das PICS ${ }^{10}$.

$\mathrm{O}$ investimento no desenvolvimento de propostas de monitoramento e avaliação (M\&A) voltadas às políticas públicas tem sido crescente, uma vez que essas são consideradas ferramentas potenciais na promoção de mudanças nas organizações e nos sistemas de saúde ${ }^{14}$. No que se refere às PICS a ampliação da sua oferta nos serviços de saúde, enquanto escolha promissora na integralidade do cuidado faz com que seja necessária a adoção de instrumentos de M\&A, como forma de qualificação do processo decisório na temática.

Diante da constatação de que há incipiência/inadequação dos sistemas de informação oficiais (ex. Sistema de Informação Ambulatorial (SIA/SUS) e Cadastro Nacional de Estabelecimentos de Saúde $(\mathrm{CNES})^{15}$, frente às necessidades peculiares às PICS no Estado em 
ISSN 2179-6750

questão, além do entendimento de que entre as funções da esfera estadual estão: a qualificação da informação, o monitoramento, e a avaliação de políticas; o Estado de Minas Gerais implantou sistema próprio de monitoramento de PICS. Tal iniciativa, a ser descrita no próximo tópico, vem sendo considerada experiência exitosa no que diz respeito ao aperfeiçoamento das ações propostas pela PEPIC-MG.

Figura 1. Divisão Administrativa de Minas Gerais-MG

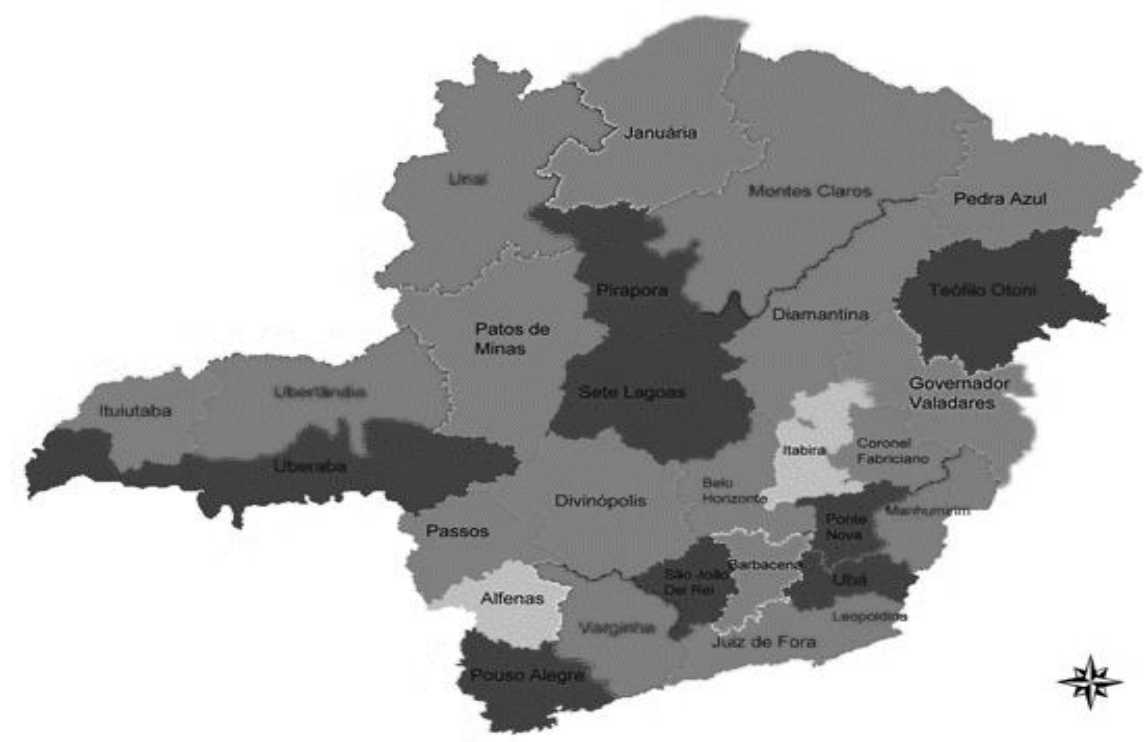

Fonte: Secretaria do Estado de Saúde Minas Gerais (SES-MG) (2016).

\section{A experiência e resultados do monitoramento da Política estadual de PICS-MG}

O monitoramento dos dados referentes aos serviços de PICS no estado é realizado por meio de um conjunto de informações recolhidas nos sistemas de informação oficiais (SIA/SUS e CNES), como também a partir de instrumento específico, desenvolvido pela CPIC, denominado de Práticas in Loco (PIL) ${ }^{6}$. O PIL começou a ser utilizado de forma experimental em 2014, e a partir do segundo trimestre de 2015, a CPIC passou a empregá-lo de forma contínua. O PIL possibilita captar informações de maneira mais flexível e dinâmica, compatível com a realidade das PICS nos municípios. Sua utilização vem permitindo compensar o sub-registro de informações no SIA-SUS, além de incluir dados sobre a oferta de plantas medicinais e fitoterapia, atualmente não contempladas no mencionado sistema.

\footnotetext{
${ }^{6}$ Minas Gerais (Estado). Práticas integrativas e complementares (PIC) [Internet]. [Belo Horizonte]: [Secretaria Estadual de Saúde]; [s.d.; citado 2018 maio 30]. Disponível em: http://www.saude.mg.gov.br/ist/page/1533-praticas-integrativase-complementares-pic
} 
ISSN 2179-6750

Cabe ressaltar, que o PIL também complementa os dados advindos do Programa de Melhoria do Acesso e da Qualidade na Atenção Básica (PMAQ-AB), uma vez que esses, quando disponíveis, vêm servindo apenas como referência da série histórica, em virtude do lapso de tempo entre a coleta, a compilação e a divulgação dos microdados (Figura 2).

Figura 2. Distribuição de municípios que declararam oferecer Práticas Integrativas e Complementares (PICS) no SUS, Minas Gerais, 2014

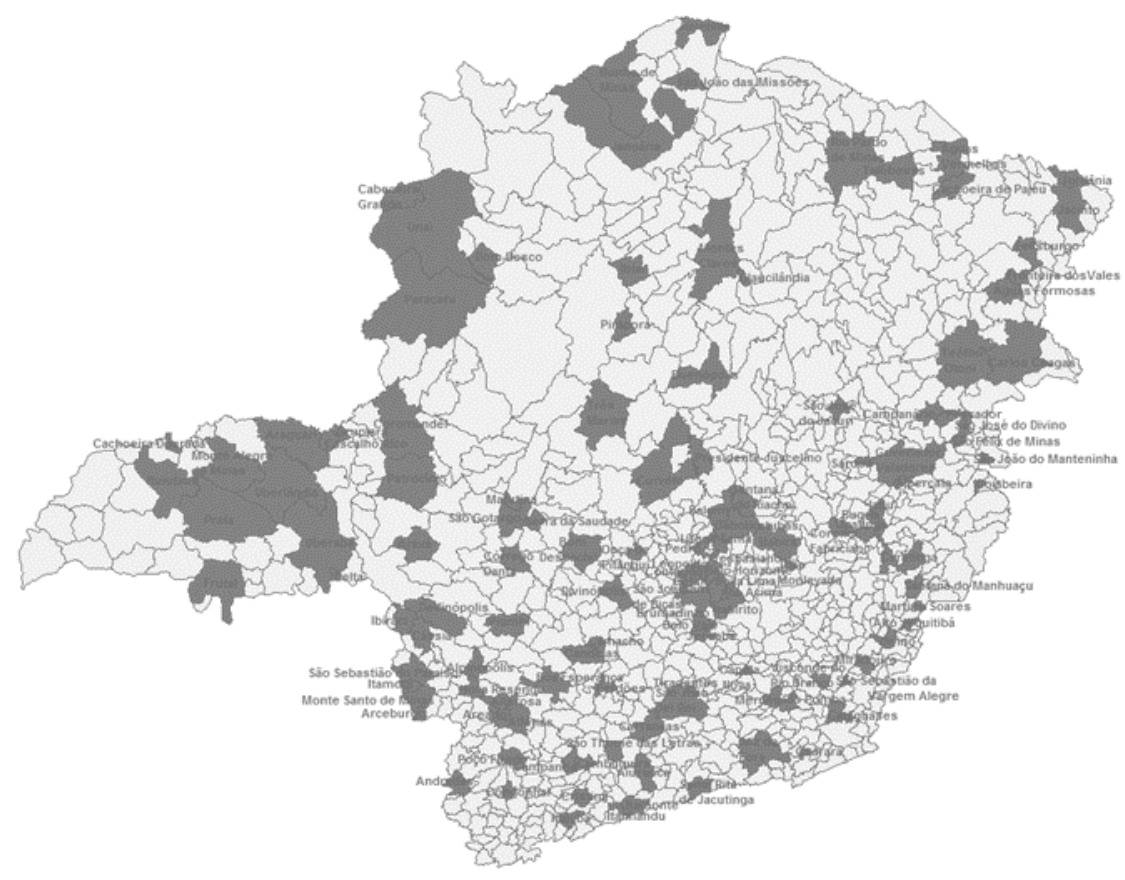

Sem registros de PIC ${ }^{\text {Total }}$ Regio
Registrou alguma PIC

Fonte: CPIC/SES-MG, Cadastro Nacional de Estabelecimentos de Saúde (CNES), Sistema de Informações Ambulatoriais do Sistema Único de Saúde (SIA/SUS) (2016).

Os indicadores a serem monitorados pelo PIL foram definidos de acordo com sua relevância e expressividade, do ponto de vista do processo de tomada de decisão, para estimar aspectos de processo e resultado. Sua definição baseou-se nos objetivos de médio e longo prazo previstos pela PEPIC, de maneira que fossem adequados/pertinentes a eles, porém com viabilidade na coleta e factibilidade na aferição. Desse modo, são monitorados: o número de procedimentos por tipo de PICS, profissional que realizou, e tipo de serviço envolvido.

O fluxo de informações do PIL compreende sua tramitação entre a CPIC, a URS e os municípios. A CPIC encaminha às URS planilha a ser preenchida pelos municípios, que as devolvem à Referência Técnica em PICS da URS, e esta reenvia para a CPIC. A coleta dos dados é mensal, mas sua sistematização é realizada trimestralmente. Tal manobra objetiva compensar oscilações na regularidade do envio. Os dados são então consolidados e utilizados, pela 
ISSN 2179-6750

Coordenação estadual, no planejamento e monitoramento das ações.

A organização do fluxo e a consolidação dos dados têm sido particularmente importantes na sua compilação e uniformização numa só área técnica estadual, de modo a facilitar seu acesso atualizado, e promovendo sua estratificação por região de saúde e por município. Isso vem proporcionando oportunidades de superação de dificuldades na implementação de sistemas de monitoramento. Para Conill ${ }^{16}$, parte dessas dificuldades se devem a profusão de formulários, relatórios, planos, dados, dificuldade de comunicação (da divulgação, retorno da informação), demandas excessivas de informações, sobrecarga das equipes locais e dificuldades de respostas concretas e implementação das mudanças.

A análise dos dados do PIL, nos anos de 2015 e 2016 aponta, de forma geral, tendência incremental no número de municípios que ofereceram e registraram a oferta de PICS, no mencionado período, com consequente aumento no número de procedimentos realizados (Gráfico $1)$.

Gráfico 1. Número de procedimentos de PICS, Minas Gerais, 2014-2016

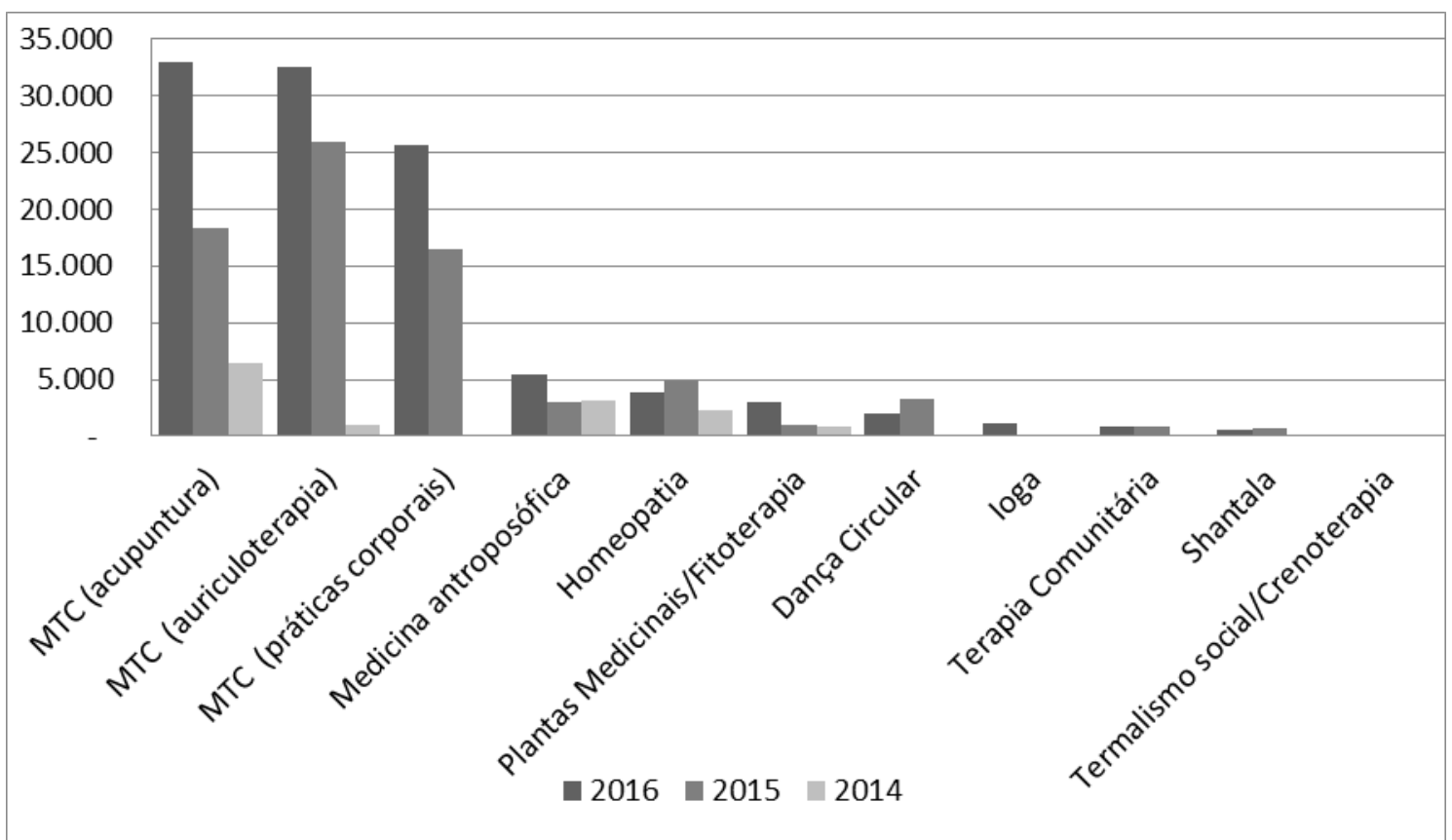

Fonte: CPIC/SES-MG (2016).

As variações observadas no período em questão podem ser devidas ao afastamento do profissional que executa determinada prática, principalmente naqueles municípios onde há só um executor. O PIL permite, também, monitorar outras práticas de cuidado, não contempladas na PEPIC-MG, inclusive algumas das práticas recém incorporadas nas tabelas de procedimentos do SIA/SUS ${ }^{17}$ (Gráficos 2 e 3 ). 
ISSN 2179-6750

Em 2015, 104 municípios registraram pelo menos um tipo de PICS no PIL, número que aumentou para 111, em 2016. Ressalta-se que não são exatamente os mesmos municípios nos dois anos, e que há municípios que registram em apenas um dos instrumentos (no SIA ou no PIL).

Gráfico 2. Outras Práticas de Cuidado ofertadas, Minas Gerais, 2014-2016

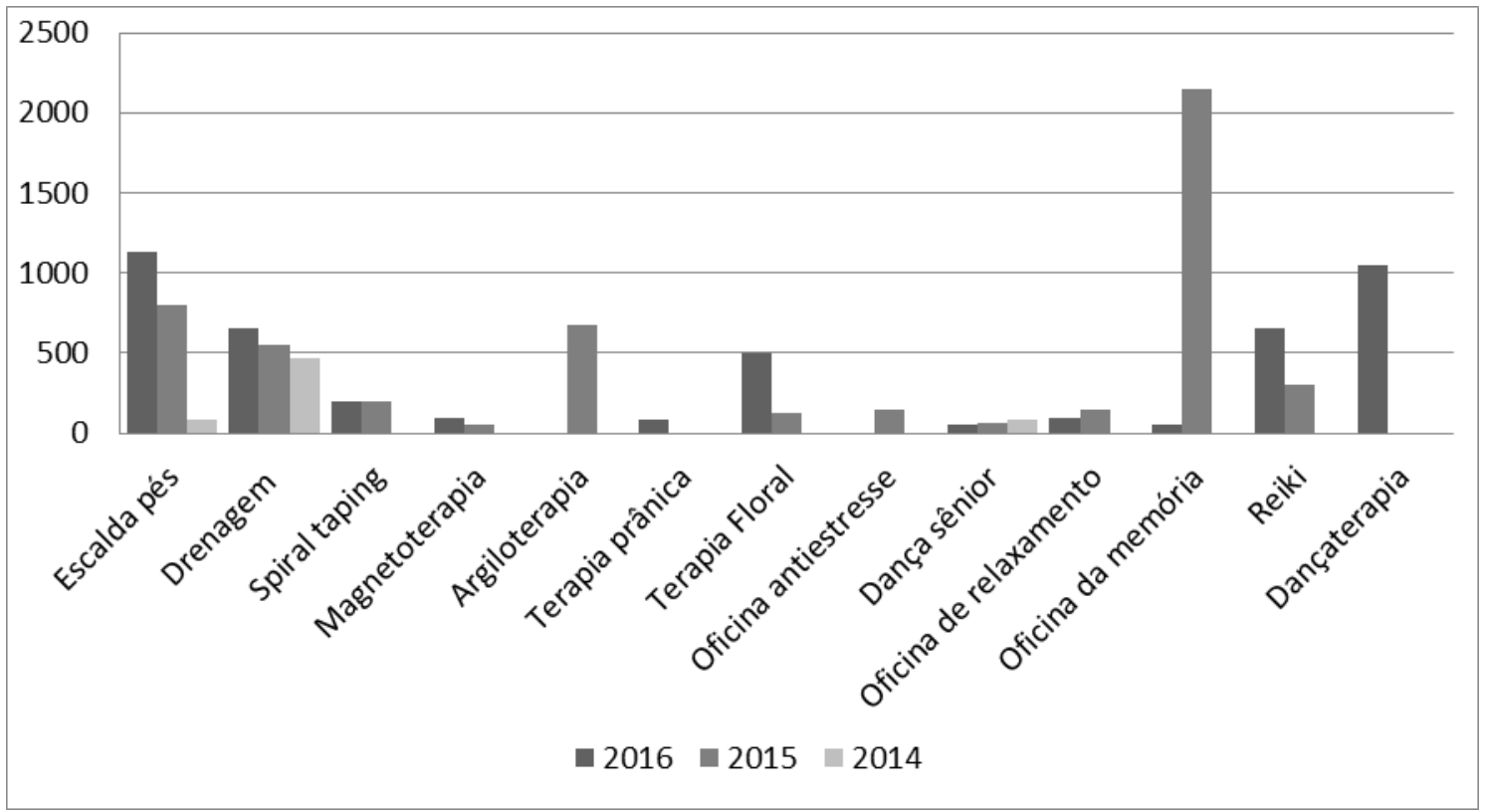

Fonte: CPIC/SES-MG (2016).

O PIL tem demonstrado ser um instrumento de apoio à gestão, pela simplicidade no manuseio, além de ser capaz de captar a diversidade das PICS e sua dinamicidade no Estado. Ademais, tem sido de grande valia na identificação de oportunidades de atuação, como também de fragilidades e dificuldades no seu alcance, facilitando o processo de tomada de decisão em tempo hábil, contribuindo na qualificação de processos de gestão, conforme recomendado por Grimm; Tanaka, $2016^{18}$.

Segundo Sousa e Tesser ${ }^{15}$, a despeito dos instrumentos normativos e dos esforços realizados, as PICS ainda não estão plenamente implantadas no País. No caso de Minas Gerais, os desafios para a consolidação da PEPIC passam pela transitoriedade da oferta das PICS, sustentada, muitas vezes, por profissionais dedicados que podem ser mais ou menos incentivados para a manutenção do serviço de acordo com o contexto sociopolítico num dado período. 
Gráfico 3. Outras Práticas de Cuidado ofertadas, Minas Gerais, 2014-2016

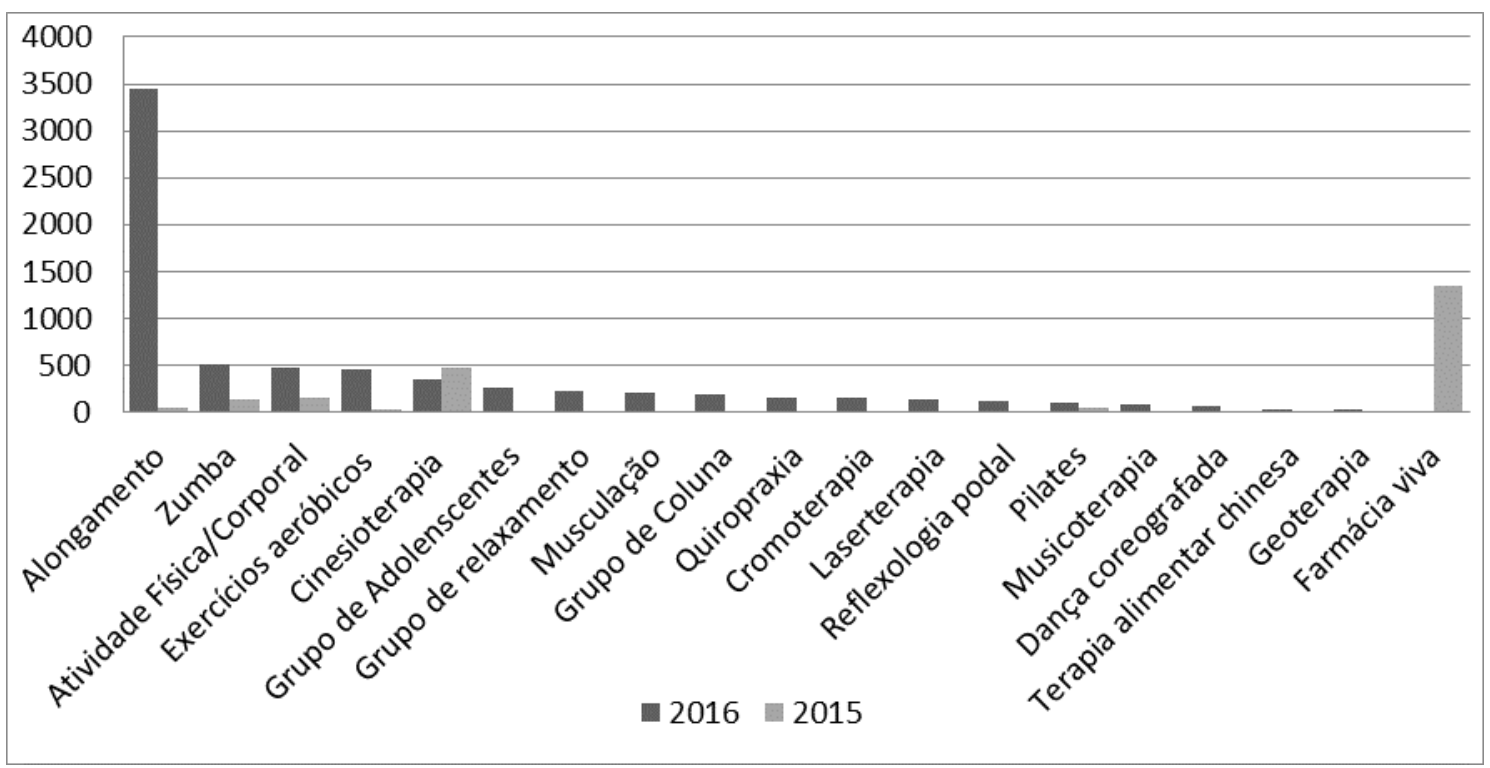

Fonte: CPIC/SES-MG (2016).

A ausência de arcabouço normativo nos municípios que institucionalizem as PICS em políticas municipais com apoio e incentivo, a falta de envolvimento de outros atores politicamente relevantes, como o controle social, os movimentos sociais e a própria população, na exigência dos seus direitos podem ser apontados como pontos a serem aprimorados para o fortalecimento das PICS.

A implementação do sistema de monitoramento objetiva analisar os desafios e potencialidades no desenvolvimento pleno do papel do gestor estadual como indutor/parceiro na formulação, na qualificação da informação, e no M\&A de políticas de PICS. De modo que o processo possa contribuir no fortalecimento de capacidades institucionais e na gestão integrada de processos.

\section{Conclusão}

A implantação de instrumento direcionado ao monitoramento das ações de PICS no Estado de Minas Gerais vem sendo importante na superação de diversas lacunas, tanto no que se refere à qualificação da informação inerente, considerada escassa e pouco adequada às necessidades requeridas no planejamento e no redirecionamento de ações nesse campo ${ }^{15}$, assim como na institucionalização dessa ferramenta no serviço público de saúde.

Reconhece-se que os serviços de saúde produzem grande quantidade de informações importantes de serem analisadas e compartilhadas de modo que possam ser úteis aos envolvidos no processo, contribuindo para o aprimoramento das políticas. No caso das PICS isso se torna 
ISSN 2179-6750

premente, tendo em conta características peculiares, como: ampla gama de práticas disponibilizadas, tidas como inovadoras, além da dinamicidade com que elas são ofertadas ${ }^{8}$.

Para Costa et al. ${ }^{19}$ as ferramentas de monitoramento ainda são pouco exploradas, em seu potencial de uso, pelas organizações de saúde, necessitando investimentos na sua implementação. Trata-se de um processo que deve der dinâmico no tempo, com envolvimento de diversos atores e utilização de diferentes métodos e competências ${ }^{20}$.

A perspectiva de monitorar intervenções complexas, a exemplo das PICS, traz contribuições significativas para a melhoria na qualidade dos serviços, ao delinear possíveis soluções e reorganizar suas atividades. Assim, produzir, sistematizar e difundir informações relacionadas a essas práticas é uma necessidade e um desafio.

No caso do monitoramento da PEPIC-MG há de se avançar na uniformização dos instrumentos utilizados com vistas a que todos os municípios que ofertem tais práticas utilizem tanto os sistemas oficiais, quanto o PIL. No que diz respeito à gestão da política destaca-se a importância do estímulo, por parte da esfera estadual, direcionado a regulamentação institucional dos programas de PICS a nível municipal. Regulamentação essa, que contemple destinação de recursos organizacionais (financeiros, humanos, materiais/físicos, entre outros), por meio de co-gestão, como maneira de favorecer a continuidade/sustentabilidade das políticas municipais de PICS ${ }^{21}$.

\section{Referências}

1. Brasil. Ministério da Saúde. Secretaria de Atenção à Saúde. Departamento de Atenção Básica. Política nacional de práticas integrativas e complementares no SUS - PNPIC-SUS. Brasília, DF: MS; 2006.92 p. (Série B. Textos básicos de saúde).

2. WHO global strategy on traditional and alternative medicine. Public Health Rep. 2002 MayJun;117(3):300-1.

3. World Health Organization. Traditional Medicine Strategy: 2014-2023. Hong Kong: WHO: 2013. $76 \mathrm{p}$.

4. Barros NF. A Construção de novos paradigmas na medicina: a medicina alternativa e a medicina complementar. In: Canesqui AM, organizador. Ciências sociais e saúde para o ensino médico. São Paulo: FAPESP; 2000. 201-13.

5. Telesi Júnior E. Práticas integrativas e complementares em saúde, uma nova eficácia para o SUS. Estud Av. 2016;30(86):99-112. http://dx.doi.org/10.1590/S0103-40142016.00100007.

6. Luz MT. Cultura contemporânea e medicinas alternativas: novos paradigmas de saúde no fim do século XX. Physis. 2005;15(Supl):145-76. 
ISSN 2179-6750

7. Tesser CD, Barros NF. Medicalização social e medicina alternativa e complementar: pluralização terapêutica do Sistema Único de Saúde. Rev Saude Publica. 2008;42(5):91420. http://dx.doi.org/10.1590/S0034-89102008000500018.

8. Tesser CD. Práticas complementares, racionalidades médicas e promoção da saúde: contribuições poucos exploradas. Cad Saude Publica. 2009;25(8):1732-42. http://dx.doi.org/10.1590/S0102-311X2009000800009.

9. Luz TM. Questões e desafios colocados para o estudo das racionalidades médicas e das práticas de saúde na atualidade: ensino, pesquisa e exercício da atenção profissional. In: Pinheiro R, Silva Junior AG, organizadores. Cidadania no cuidado: o universal e o comum na integralidade das ações em saúde. Rio de Janeiro: IMS/UERJ-CEPESC; 2011, p. 295305 .

10. Minas Gerais. Secretaria de Estado de Saúde de Minas Gerais. Política estadual de práticas integrativas e complementares. Belo Horizonte: Secretaria Estadual de Saúde; 2009.

11. Minas Gerais. Escola de Saúde Pública do Estado de Minas Gerais. Secretaria de Estado de Saúde de Minas Gerais. Subsecretaria de Políticas e Ações de Saúde. Caderno de práticas integrativas e complementares no SUS/MG: formação de apoiadores, experiências e conceitos. Belo Horizonte: Escola de Saúde Pública do Estado de Saúde de Minas Gerais; 2014.

12. Prefeitura Municipal de Belo Horizonte. Secretaria Municipal de Saúde. PRHOAMA: programa de homeopatia, acupuntura e medicina antroposófica no SUS-BH. Belo Horizonte: Secretaria Municipal de Saúde; 2004.

13. Prefeitura Municipal de Belo Horizonte. Secretaria Municipal de Saúde. PRHOAMA: programa de homeopatia, acupuntura e medicina antroposófica 21 anos: um verdadeiro encontro com a saúde. Belo Horizonte: Secretaria Municipal de Saúde; 2015.

14. Denis JL. Institucionalização da avaliação na administração pública. Rev Bras Saude Matern Infant. 2010;10(Supl 1):S229-S237. http://dx.doi.org/10.1590/S1519-38292010000500020.

15. Sousa IMC, Tesser CD. Medicina Tradicional e Complementar no Brasil: inserção no Sistema Único de Saúde e integração com a atenção primária. Cad Saude Publica. 2017;33(1):1-15. http://dx.doi.org/10.1590/0102-311X00150215.

16. Conill EM. Sobre a importância da parcimônia, do diálogo e da continuidade para a institucionalização da avaliação da atenção básica. Cienc Saude Colet. 2006;11(3):564-76.

17. Ministério da Saúde (BR). Portaria no. 145 de 11 de janeiro de 2017. Altera procedimentos na tabela de procedimentos, medicamentos, órteses, próteses e materiais especiais do SUS para atendimento na atenção básica. Diário Oficial da União. 2017 jan 11; Seção 1(10):32. 
ISSN 2179-6750

18. Grimm SCA, Tanaka OY. Painel de monitoramento municipal: bases para a construção de um instrumento de gestão dos serviços de saúde. Epidemiol Serv Saude. 2016;25(3):585-94. http://dx.doi.org/10.5123/s1679-49742016000300014.

19. Costa JMBS, Felisberto E, Bezerra LCA, Cesse EÂP, Samico IC. Monitoramento do desempenho da gestão da vigilância em saúde: instrumento e estratégias de uso. Cienc Saude Colet. 2013;18(5):1201-16.

20. Figueiró AC, Frias PG, Navarro LM. Avaliação em saúde: conceitos básicos para a prática nas instituições. In: Samico I, Felisberto E, Figueiró AC, Frias PG, organizadores. Avaliação em saúde: bases conceituais e operacionais. Rio de Janeiro: MedBook, 2010. p. 1-14.

21. Pluye P, Potvin L, Denis J-L, Pelletier J, Mannoni C. Program sustainability begins with the first events. Eval Program Plan. 2005;(28):123-37. https://doi.org/10.1016/j.evalprogplan.2004.10.003. 